\title{
Heterogeneous Nucleation in and out of Pores
}

\author{
Amanda J. Page* and Richard P. Sear ${ }^{\dagger}$ \\ Department of Physics, University of Surrey, Guildford, Surrey GU2 7XH, United Kingdom
}

(Received 2 May 2006; published 10 August 2006)

\begin{abstract}
We study the nucleation of a new thermodynamic phase in pores and find that the nucleation often proceeds via two steps: nucleation of pore filling, and nucleation out of the pore. These two rates have opposing dependencies on pore size, resulting in a pore size at which the nucleation rate of the new phase is maximal. This finding is relevant to attempts to design and use porous media to crystallize proteins.
\end{abstract}

PACS numbers: 64.60.Qb, 68.55.Ac, 82.60.Nh

Crystallization and condensation both start with heterogeneous nucleation, which occurs at a surface. The nucleus of the crystal or liquid phase forms in contact with this surface. In both cases nucleation is an activated process [1]. The barrier to formation and hence the rate of crystallization or condensation depends on the properties of the surface. In particular, this rate is known to be very sensitive to the geometry of the surface. Studies of nucleation in pores where the surfaces of the pore may confine the nucleus along one or more directions [2-5] have found that both the size and shape of the pore strongly effect the nucleation rate. These studies were motivated by the desire to understand the hysteresis of phase transitions inside porous media [6,7]. Here, we study nucleation in pores via computer simulations, and we find that nucleation occurs in the corners of pores. Thus, for example, unless a slit pore is very long we expect condensation in it to occur via nucleation in a corner, not via nucleation far from the pore ends as was studied in earlier work [2,3]. This is consistent with the work of Paul and Rieger [7] who found less hysteresis for a closed pore (which has corners) than for an open pore (without corners).

Thus our work is relevant to condensation in porous media. However, the primary motivation behind this work is to better understand the nucleation of protein crystals in solutions that contain a piece of porous medium $[8,9]$. The nucleation only occurs in the presence of the porous medium and the crystals are found to be stuck to this medium, implying that the protein crystal nucleates on the surface of the porous medium. Surfaces that are not porous have proved less successful at promoting nucleation $[9,10]$. This is evidence that it is the geometry of the pores that is accelerating nucleation. Finally, only disordered porous media were effective; media in which the pores were of uniform size, such as zeolites, did not induce nucleation $[9,10]$. It was hypothesized $[8,9]$ that there is a pore size at which the nucleation rate was maximal and that as disordered porous media have pores with a range of sizes a disordered porous medium is likely to have pores near this size. A zeolite's pores are all the same size and it is improbable that this size will just happen to be a size at which the nucleation is fast. We find here that there is indeed a pore size at which the nucleation rate is fastest, supporting the earlier hypothesis.

As we are interested in generic features of nucleation from pores, we study perhaps the simplest model: the twodimensional Ising model on a square lattice with nearestneighbor interactions [11,12]. This has previously been used to study heterogeneous nucleation $[13,14]$. As before [14] we study the model using Monte Carlo simulations [11]. Each site $i$ on the square lattice has a spin $s_{i}= \pm 1$ associated with it. In the Ising model at low temperatures there is a transition from a phase with predominantly down, -1 , spins to one with predominantly up, +1 , spins. We will always study nucleation of the spin-up phase from the spindown phase. The surface is formed of a region where the spins are fixed so that the spin $s_{i}=+1$. The rest of the spins we refer to as free spins. The experimental work which inspires our calculations is all on either crystals [9] or fluids [6] and porous media, yet we simulate a simple model magnet. We do so because nucleation is expected to depend weakly on whether the dynamics are spin-flips or particle diffusion.

The energy $E$ of the system consists of three sums,

$$
E=J \sum_{i j}^{\prime} s_{i} s_{j}-h \sum_{i} s_{i}+J_{s} \sum_{i j}^{\prime \prime} s_{i} s_{j},
$$

where the first term is from interactions between free spins, the second is the interaction between free spins and an external magnetic field $h$, and the third is for the interaction between the free spins and the fixed spins of the wall. The dash on the first sum indicates that it is over all nearestneighbor pairs of free spins, and the double dash over the last sum indicates that it is over all nearest-neighbor pairs of free and fixed spins. In Eq. (1), $J$ is the strength of the coupling between free spins and $J_{s}$ is the strength of the coupling between a free spin and a fixed spin of the surface. Here we consider only $J_{s}=0$. Our surfaces do not attract either phase. The surface free energies for the surface-spinup, and surface-spin-down phases are identical and a spinup-spin-down interface hits the surface at a contact angle $\theta=90^{\circ}$.

The pores we study all have a simple rectangular slit pore geometry; see Fig. 1. The surfaces of the sides and 


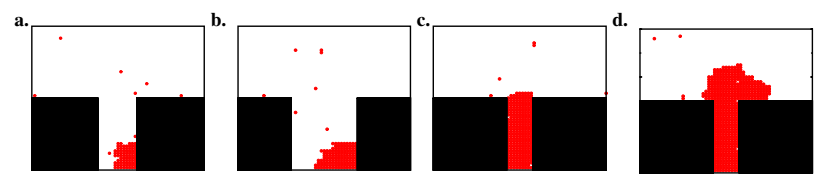

FIG. 1 (color online). Simulation snapshots of systems 60 by 60 sites in size at $J / k T=0.8, h / k T=0.05$. All pores are 30 sites deep and have fixed spins along both sides and the bottom. The fixed spins are black, while the up spins are red. The sites with down spins are left white. The pores in (a), (b), (c), and (d) are 13, 24, 9, and 9 sites wide, respectively. All except (c) show a nucleus near the top of the barrier, for example, when we started 100 runs from the configuration of snapshot (a), 47 of these configurations resulted in a full pore, the rest resulted in the nucleus shrinking to nothing. Snapshot (c) shows a typical configuration for a pore filled with the spin-up phase but with the bulk in the spin-down phase.

bottom of the pores are composed of fixed spins. Simulating the nucleation rates directly is prohibitively slow if the rates are low. Therefore, we will use the forward flux sampling (FFS) algorithm of Allen and coworkers [15]. This has previously been used to study nucleation in the Ising model [14] and also in an off-lattice model [16]. This allows us to efficiently calculate very low nucleation rates. For example, for the purposes of comparison we calculated the rate of homogeneous nucleation and found it to be $8.4 \times 10^{-26} \pm 2.1 \times 10^{-26} / \mathrm{cycle} / \mathrm{site}$. One cycle is one attempted spin flip per lattice site. Homogeneous nucleation is nucleation in the bulk; the nucleus forms far from any surface. This is at $J / k T=0.8$ and $h / k T=0.05$. The critical point of the two-dimensional Ising model is at $J / k T=0.44$ [12], so we are far below the critical temperature. For the same values of $J$ and $h$, we also calculated the rate of heterogeneous nucleation on a perfectly flat surface. It is $3.7 \times 10^{-14} \pm 1.2 \times$ $10^{-14} /$ cycle/surface site. Per site the nucleation rate is 12 orders of magnitude faster on a smooth surface than in the bulk. Thus for a system bounded by a smooth wall heterogeneous nucleation dominates unless the system is more than $10^{12}$ sites across.

We will now consider nucleation from pores. The principle results of our simulations are that (i) nucleation is orders of magnitude faster at the pores than on a smooth surface, and (ii) that it often proceeds via two steps. The second observation is clearly seen in Fig. 2. This shows the number of up spins as a function of time, for a system that is initially in the spin-down phase. The system clearly remains stable in the spin-down phase for approximately 12000 cycles before the number of up spins rapidly increases to around 200 where it remains for a little more than 20000 cycles, before increasing again. In both cases the number of spins is stable over thousands of cycles due to the existence of a nucleation barrier. It is kept stable close to zero by a nucleation barrier for pore filling, then it is kept stable at around 200 by a barrier to nucleation out of

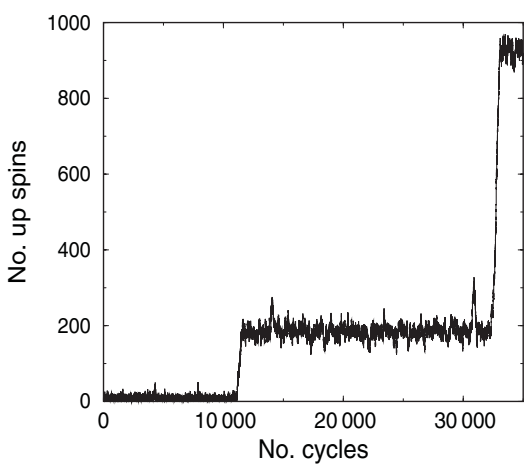

FIG. 2 (color online). The number of up spins, as a function of time, in a system of 40 by 40 lattice sites with a pore 9 sites across and 20 deep. The system starts in the spin-down phase, and $J / k T=0.7$ and $h / k T=0.05$.

a pore. Between 12000 and 32000 cycles the pore is full but in the bulk the system is still in the spin-down phase; see Fig. 1(c) for a typical configuration.

The FFS algorithm of Allen and coworkers [15] calculates the rate of a one-step activated process whereas here we sometimes have a two-step process. However, the total mean time for nucleation at a pore, $\tau_{N}$, is simply the sum of the mean time to nucleate inside a pore, $\tau_{\mathrm{FILL}}$, and the mean time to nucleate out of a pore, $\tau_{\mathrm{OUT}}$, if both nucleation times are long, much longer than any times for growth. Thus, if we want the nucleation rate for the spin-up phase in the presence of a pore $\alpha_{N}=\tau_{N}^{-1}$ it is simply given by

$$
\alpha_{N}=\left(\alpha_{\mathrm{FILL}}^{-1}+\alpha_{\mathrm{OUT}}^{-1}\right)^{-1} \text {, }
$$

where $\alpha_{\mathrm{FILL}}=\tau_{\mathrm{FILL}}^{-1}$ and $\alpha_{\mathrm{OUT}}=\tau_{\mathrm{OUT}}^{-1}$. Thus we use the FFS algorithm to calculate the individual rates $\alpha_{\text {FILL }}$ and $\alpha_{\text {OUT }}$ then Eq. (2) to calculate the desired total rate. As an order parameter the total number of up spins was used, although for nucleation in the pore we also tried using the number of up spins in the pore and found that it gave the same results but was no more efficient. Note that the FFS algorithm is only weakly sensitive to the choice of order parameter [15].

The rate of nucleation of the bulk spin-up phase, as a function of the pore width $w$, is plotted in Fig. 3(a). The rates for nucleation in the pore, $\alpha_{\mathrm{FILL}}$, and out of the pore, $\alpha_{\text {OUT }}$, are shown in Fig. 3(b). This is for $J / k T=0.8$ and $h / k T=0.05$. Data for $J / k T=0.7$ (not shown) show the same features, including the peak. For small $w$, as $w$ decreases the rate tends to the rate for a flat surface, of order $10^{-14}$, while for large $w$, it reaches a plateau. There is a clear maximum in the rate for pores $w=12$ and 13 lattice sites across. Thus if, as in attempts to use porous media to induce the nucleation of protein crystals [9], it is desired to maximize the nucleation rate, there is an optimum pore width. The results of Fig. 3 are for pores of depth 30 . We determined that this was sufficiently deep to ensure that the nucleation rates were essentially independent of 
a.

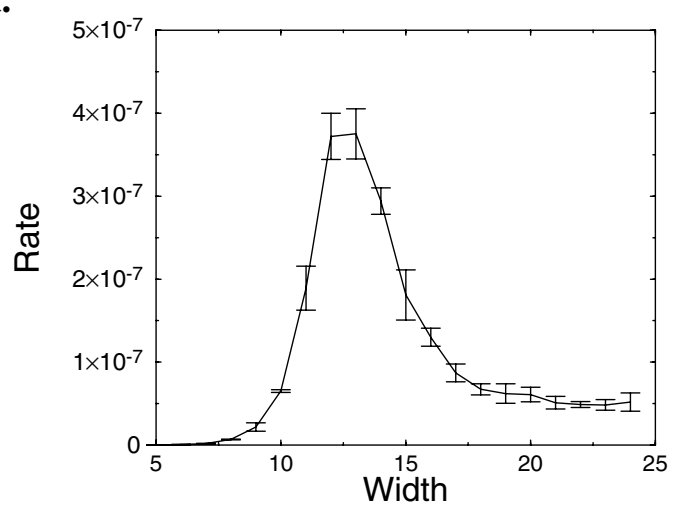

b.

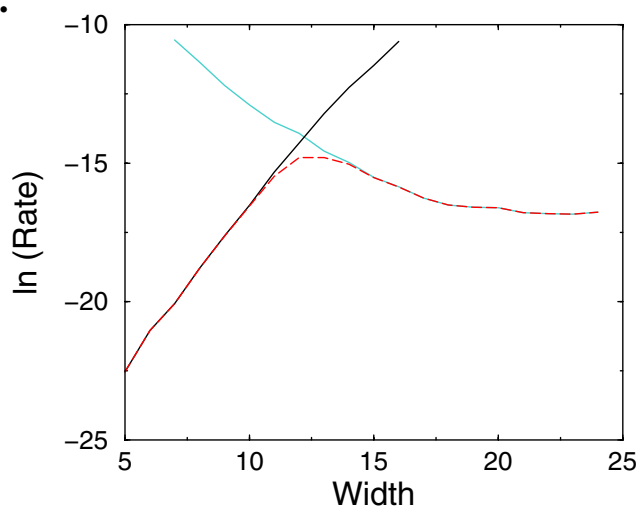

FIG. 3 (color online). (a) the rate of nucleation as a function of pore width $w$. The system is of 60 by 60 lattice sites, the pore is 30 sites deep, $J / k T=0.8$ and $h / k T=0.05$. The error bars are the standard deviations of the rates obtained in 4 independent runs. (b) $\ln$ (Rate), as a function of pore width $w$, for the same system. The dashed curve (red online) is the overall rate for nucleation from a pore, the light gray (cyan online) curve is the nucleation rate in a pore, and the black curve is the nucleation rate of the bulk spin-down phase out of a pore that is already filled with the spin-up phase.

depth. For example, for pores of width 12 and depth 20 the calculated nucleation rate is $8.1 \times 10^{-7} \pm 1.4 \times$ $10^{-7} /$ cycle while if the depth is 30 the rate is $9.0 \times$ $10^{-7} \pm 0.7 \times 10^{-7} /$ cycle. To help us understand the dependence of the rate on the pore width $w$ we turn to classical nucleation theory (CNT).

CNT [1] approximates the nucleation rate by

$$
\text { Rate }=\nu \exp \left(-\Delta F^{*} / k T\right),
$$

where $\Delta F^{*}$ is the height of the free-energy barrier to nucleation, the free energy of the critical nucleus, and $\nu$ is an attempt frequency. We take $\nu=1 /$ cycle. Now for homogeneous nucleation in the two-dimensional Ising model, the free energy of a circular nucleus of radius $R$ is

$$
\Delta F=-2 \pi R^{2} h+2 \pi R \gamma,
$$

where the first term is the bulk term, the gain in free energy from forming the lower free-energy spin-up phase, and the second term is the surface term, the free energy cost of forming a spin-up-spin-down interface at the edge of the nucleus. The interfacial tension between the two phases $\gamma$ is given by Onsager's [12] exact expression and is $\gamma=$ $1.19 k T$ at $J / k T=0.8$. The maximum in $\Delta F$ of Eq. (4), $\Delta F_{\mathrm{HOMO}}^{*}=\pi \gamma^{2} /(2 h)$, and occurs for a critical nucleus of radius $R^{*}=\gamma /(2 h)$.

Aside from homogeneous nucleation we will use CNT to estimate the nucleation rates for nucleation on a perfectly smooth surface, nucleation in a corner, and nucleation on top of a filled pore. For simulation snapshots of nucleation in a corner and on top of a filled pore see Figs. 1(b) and 1(d), respectively. For heterogeneous nucleation on a perfectly flat surface the nucleus is a semicircle because the contact angle between spin-up-spin-down interface and the surface is $\theta=90^{\circ}$. The nucleus is just half the nucleus for homogeneous nucleation and so has half the free energy. Thus the nucleation barrier on a smooth surface $\Delta F_{\mathrm{FLAT}}^{*}=\Delta F_{\mathrm{HOMO}}^{*} / 2$, while the critical radius is the same, $R^{*}$. For a wide pore, nucleation occurs in a $90^{\circ}$ corner and the critical nucleus is too small to feel the effect of the other corner of the pore; see Fig. 1(b). There the nucleus is a quarter circle, and so the nucleation barrier in a right-angle corner $\Delta F_{90}^{*}=\Delta F_{\mathrm{HOMO}}^{*} / 4$, with again a radius $R^{*}$.

So, within CNT the free-energy barriers for homogeneous nucleation, nucleation on a perfectly smooth surface and nucleation in a corner are $\Delta F_{\mathrm{HOMO}}^{*}, \Delta F_{\mathrm{HOMO}}^{*} / 2$, and $\Delta F_{\mathrm{HOMO}}^{*} / 4$, respectively. Then $\mathrm{CNT}$ predicts that $\ln \left(\right.$ Rate $\left._{\text {HOMO }} / \nu\right) / \ln \left(\right.$ Rate $\left._{\text {FLAT }} / \nu\right)=0.5$ and $\ln \left(\right.$ Rate $\left._{90} / \nu\right) /$ $\ln \left(\operatorname{Rate}_{\text {номо }} / \nu\right)=0.25$. As we have calculated all three rates from simulation we can determine the actual ratios and they are 0.54 and 0.30 , respectively. Given the simple nature of CNT the agreement is satisfactory.

The final nucleation barrier we need to calculate is for nucleation out of an already filled pore. A snapshot of the spin-up phase nucleating out of a pore is shown in Fig. 1(d). As the pore is already filled before nucleation occurs, this is not part of the nucleus. The CNT nucleus is a semicircle as for nucleation on a perfectly smooth surface. Thus the area, and hence the bulk term in the free energy is as for nucleation on a flat surface. However, as we can see from the simulation snapshot of Fig. 1(d), along its base the nucleus is in contact for $w$ spins not with the surface but with the spin-up phase that fills the pore. This contributes a term $-w \gamma$ to the free energy of formation of the nucleus. So for nucleation out of a pore the free-energy barrier is

$$
\Delta F_{\mathrm{OUT}}^{*}=\Delta F_{\mathrm{HOMO}}^{*} / 2-w \gamma, \quad 2 R^{*} \geq w .
$$

Nucleation out of a filled pore is always faster than on a perfectly smooth surface, and the log of the rate should vary linearly with $w$. In Fig. 3(b) we have plotted the log of the rate of nucleation out of a pore as the black curve. We see that indeed it varies linearly with $w$; also the slope, at 1.09 , is close to $\gamma / k T=1.19$. 
Thus the rate of nucleation out of the pore increases exponentially with $w$. The rate of nucleation of pore filling has the opposite dependence on $w$. For large $w$ nucleation of pore filling occurs with a nucleus in one corner that is much less than $w$ spins across and so does not interact with the opposite side of the pore. This then leads to the plateau where the nucleation rate is independent of $w$. However, for $w$ comparable to the nucleus size $R^{*}$ the nucleus does feel the effect of the opposite side. CNT gives $R^{*}=12$ at the conditions of Fig. 3. In Fig. 1(a) we have plotted a nearcritical nucleus for a pore of width 13. Note that it is smaller than the nucleus in a wide pore, Fig. 1(b). The nucleus has been displaced to smaller sizes as by the time that it has grown to span the pore it is over the barrier. This smaller critical nucleus has a smaller free-energy barrier and hence the rate of pore filling is larger for pores of widths $\sim R^{*}$ than for wider pores. At the values of $w$ around $R^{*}$ nucleation inside the pore is still rate limiting and so this increase leads directly to an increase in nucleation rate of the bulk phase and hence to the maximum in the nucleation rate as a function of $w$. We have performed simulations at other values of $J$ and $h$, and we also have preliminary results for $J_{s} \neq 0$ and for a different pore geometry, a wedge. In these systems too we have found maxima in the rate; thus we are confident that a nonmonotonic variation in the rate with pore width is not restricted to our particular pore geometry and value for $J_{s}$.

Nucleation in pores 12 to 13 sites across is 7 orders of magnitude faster than on a flat surface. Therefore, if these pores are present at a density of more than one pore per million lattice sites of the surface, then nucleation from these pores will dominate nucleation on the smooth parts of the surface. Even at very low densities of pores nucleation at them dominates. Most nucleation, e.g., of ice in our freezers, is heterogeneous and occurs on dirt or a container surface. These surfaces will not be atomically smooth. The critical nucleus for ice will be approximately a nanometer across and we would expect the surfaces of dirt to have many indentations, which will resemble pores, a nanometer or more deep. Thus, we would like to suggest that for water and for other molecular systems, new phases often nucleate in small indentations on surfaces, not on smooth parts of the surface. This is not the first suggestion that pores are important; over 50 years ago Turnbull [17] suggested that the history-dependent nucleation found in some transitions, such as the crystallization of gallium, was due to the nucleation occurring in pores.

We have considered heterogeneous nucleation at pores. We found: (i) that the nucleation rate is orders of magnitude higher at pores that on flat surfaces, (ii) that this rate is very sensitive to size of the pore-we found that the rate had a maximum when our pores were 12 to 13 lattice sites across - and (iii) that for some pore widths the new bulk phase forms via two successive activated processes. The two processes are nucleation of pore filling and nucleation out of the pore to form the bulk spin-up phase. Either one can be rate limiting. Within our Ising model the smaller the pore the faster was pore filling but the slower was nucleation out of the pore. Thus to maximize the nucleation rate of a protein crystal, the pores should not be much smaller than the size of the critical nucleus of the crystal, as then nucleation out of the pore is slow, nor should they be much larger than the critical nucleus, as then the initial pore filling is slow. A pore approximately the same size as the critical nucleus is optimal.

The authors would like to thank the EPSRC for financial support to A.J.P.

*Electronic address: a.page@ surrey.ac.uk

${ }^{\dagger}$ Electronic address: r.sear@surrey.ac.uk

[1] P. G. Debenedetti, Metastable Liquids (Princeton University Press, Princeton, 1996).

[2] F. Restagno, L. Bocquet, and T. Biben, Phys. Rev. Lett. 84, 2433 (2000).

[3] V. Talanquer and D. W. Oxtoby, J. Chem. Phys. 114, 2793 (2001).

[4] B. Husowitz and V. Talanquer, J. Chem. Phys. 121, 8021 (2004).

[5] A. Saugey, L. Bocquet, and J. L. Barrat, J. Phys. Chem. B 109, $6520(2005)$

[6] L.D. Gelb, K.E. Gubbins, R. Radhakrishnan, and M. Sliwinska-Bartkowiak, Rep. Prog. Phys. 62, 1573 (1999).

[7] R. Paul and H. Rieger, J. Chem. Phys. 123, 024708 (2005).

[8] N.E. Chayen, E. Saridakis, R. El-Bahar, and Y. Nemirovsky, J. Mol. Biol. 312, 591 (2001).

[9] N.E. Chayen, E. Saridakis, and R. P. Sear, Proc. Natl. Acad. Sci. U.S.A. 103, 597 (2006).

[10] N. E. Chayen and E. Saridakis, J. Cryst. Growth 232, 262 (2001).

[11] D. Chandler, Introduction to Modern Statistical Mechanics (Oxford University Press, New York, 1987).

[12] L. Onsager, Phys. Rev. 65, 117 (1944).

[13] E. N. M. Cirillo and J. L. Lebowitz, J. Stat. Phys. 90, 211 (1998).

[14] R. P. Sear, J. Phys. Chem. B 110, 4985 (2006).

[15] R. J. Allen, P. B. Warren, and P. R. ten Wolde, Phys. Rev. Lett. 94, 018104 (2005); R. J. Allen, D. Frenkel, and P. R. ten Wolde, J. Chem. Phys. 124, 024102 (2006).

[16] C. Valariani, E. Sanz, and D. Frenkel, J. Chem. Phys. 122, 194501 (2005).

[17] D. Turnbull, J. Chem. Phys. 18, 198 (1950). 